\title{
Correction to: Effectiveness of guideline dissemination and implementation strategies on health care professionals' behaviour and patient outcomes in the cancer care context: a systematic review
}

\author{
Jennifer R. Tomasone ${ }^{1 *}$, Kaitlyn D. Kauffeldt ${ }^{1}$, Rushil Chaudhary ${ }^{2}$ and Melissa C. Brouwers ${ }^{3}$
}

Correction to: Implementation Sci 15, 41 (2020)

https://doi.org/10.1186/s13012-020-0971-6

Following the publication of the original article [1], it was reported that there was an error in the order and content of Tables 2-4. The correct Tables 2, 3, 4 are given in this Correction article.

The publisher apologizes to the authors and readers for the inconvenience.

\footnotetext{
Author details

${ }^{1}$ School of Kinesiology \& Health Studies, Queen's University, 28 Division Street, Kingston, Ontario, Canada. ${ }^{2}$ Department of Medicine, University of Toronto, 27 King's College Circle, Toronto, Ontario, Canada. ${ }^{3}$ School of Epidemiology and Public Health, University of Ottawa, 600 Peter Morand Crescent, Ottawa, Ontario, Canada.
}

Published online: 02 June 2021

\footnotetext{
Reference

1. Tomasone JR, Kauffeldt KD, Chaudhary R, Brouwers MC. Effectiveness of guideline dissemination and implementation strategies on health care professionals' behaviour and patient outcomes in the cancer care context: a systematic review. Implementation Sci. 2020;15:41 https://doi.org/10.1186/ s13012-020-0971-6.
}

The original article can be found online at https://doi.org/10.1186/s13012020-0971-6

* Correspondence: tomasone@queensu.ca

${ }^{1}$ School of Kinesiology \& Health Studies, Queen's University, 28 Division Street, Kingston, Ontario, Canada

Full list of author information is available at the end of the article

(C) The Author(s). 2021 Open Access This article is licensed under a Creative Commons Attribution 4.0 International License, which permits use, sharing, adaptation, distribution and reproduction in any medium or format, as long as you give appropriate credit to the original author(s) and the source, provide a link to the Creative Commons licence, and indicate if changes were made. The images or other third party material in this article are included in the article's Creative Commons licence, unless indicated otherwise in a credit line to the material. If material is not included in the article's Creative Commons licence and your intended use is not permitted by statutory regulation or exceeds the permitted use, you will need to obtain permission directly from the copyright holder. To view a copy of this licence, visit http://creativecommons.org/licenses/by/4.0/. The Creative Commons Public Domain Dedication waiver (http://creativecommons.org/publicdomain/zero/1.0/) applies to the data made available in this article, unless otherwise stated in a credit line to the data. 
Table 2 Risk of bias for randomized controlled trials

\begin{tabular}{|c|c|c|c|c|c|c|c|}
\hline & 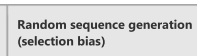 & 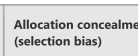 & 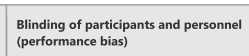 & 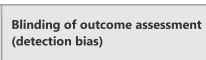 & 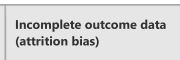 & 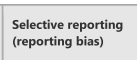 & 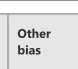 \\
\hline Apyestat:[28] & + & $?$ & $?$ & $?$ & $\odot$ & $?$ & $?$ \\
\hline 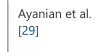 & + & $?$ & + & + & + & + & $?$ \\
\hline 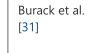 & $\odot$ & $?$ & $?$ & $?$ & $\odot$ & $?$ & $?$ \\
\hline $\begin{array}{l}\text { camayy atal } \\
\text { Barl }\end{array}$ & $?$ & $?$ & $?$ & $?$ & + & $?$ & $?$ \\
\hline 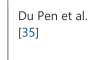 & $\odot$ & $?$ & $?$ & $?$ & $\odot$ & $\odot$ & $?$ \\
\hline 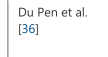 & $\odot$ & $?$ & $?$ & $\odot$ & + & + & $?$ \\
\hline 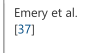 & $\odot$ & $\odot$ & $?$ & $?$ & $?$ & $?$ & $?$ \\
\hline 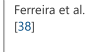 & $?$ & $?$ & $?$ & $?$ & + & + & $?$ \\
\hline Concetat [159] & ? & ? & $?$ & $?$ & + & $?$ & $?$ \\
\hline 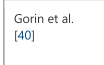 & $\odot$ & $?$ & $?$ & $\odot$ & 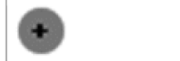 & $?$ & $?$ \\
\hline 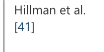 & $\odot$ & $?$ & $?$ & $?$ & $\odot$ & $?$ & $?$ \\
\hline 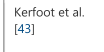 & $\odot$ & $?$ & $?$ & $?$ & + & + & $?$ \\
\hline 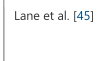 & + & $?$ & $?$ & $\odot$ & + & 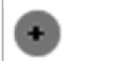 & $?$ \\
\hline tingetal: [4] & $\odot$ & $\odot$ & $?$ & $\odot$ & + & $?$ & $?$ \\
\hline 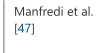 & + & $?$ & $?$ & $\odot$ & $\odot$ & $\odot$ & $?$ \\
\hline 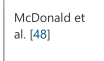 & $?$ & $\odot$ & $?$ & $\odot$ & $\odot$ & $\odot$ & $?$ \\
\hline $\begin{array}{l}\text { Mereseat } \\
\text { kat }\end{array}$ & + & $?$ & $?$ & $?$ & + & + & $?$ \\
\hline $\begin{array}{l}\text { Meres at } \\
\text { Daf }\end{array}$ & $?$ & $?$ & $?$ & $?$ & $?$ & $?$ & $?$ \\
\hline 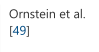 & + & $?$ & $?$ & $?$ & + & + & $?$ \\
\hline Patetal|[s] & + & $\odot$ & - & $?$ & + & $\odot$ & $?$ \\
\hline Rolue ata [56] & $?$ & $?$ & $\odot$ & $?$ & $\odot$ & $?$ & $?$ \\
\hline $\begin{array}{l}\text { sisustetat } \\
\text { sint }\end{array}$ & + & $?$ & $?$ & $?$ & + & + & $?$ \\
\hline $\begin{array}{l}\text { wasthe thal } \\
\text { sist }\end{array}$ & + & $?$ & $?$ & $?$ & + & $?$ & $?$ \\
\hline 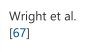 & + & $?$ & $?$ & $?$ & + & $?$ & $?$ \\
\hline
\end{tabular}




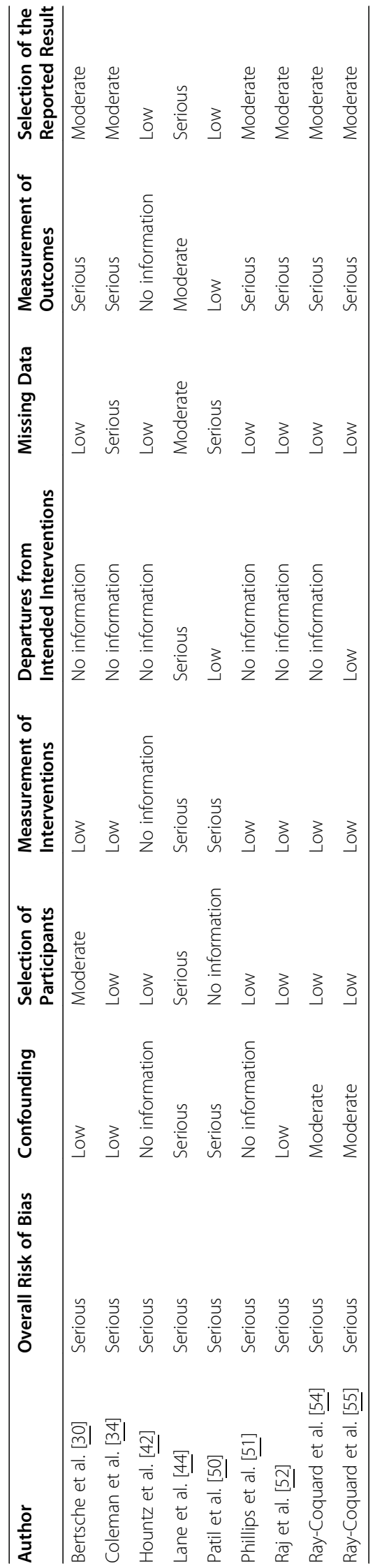




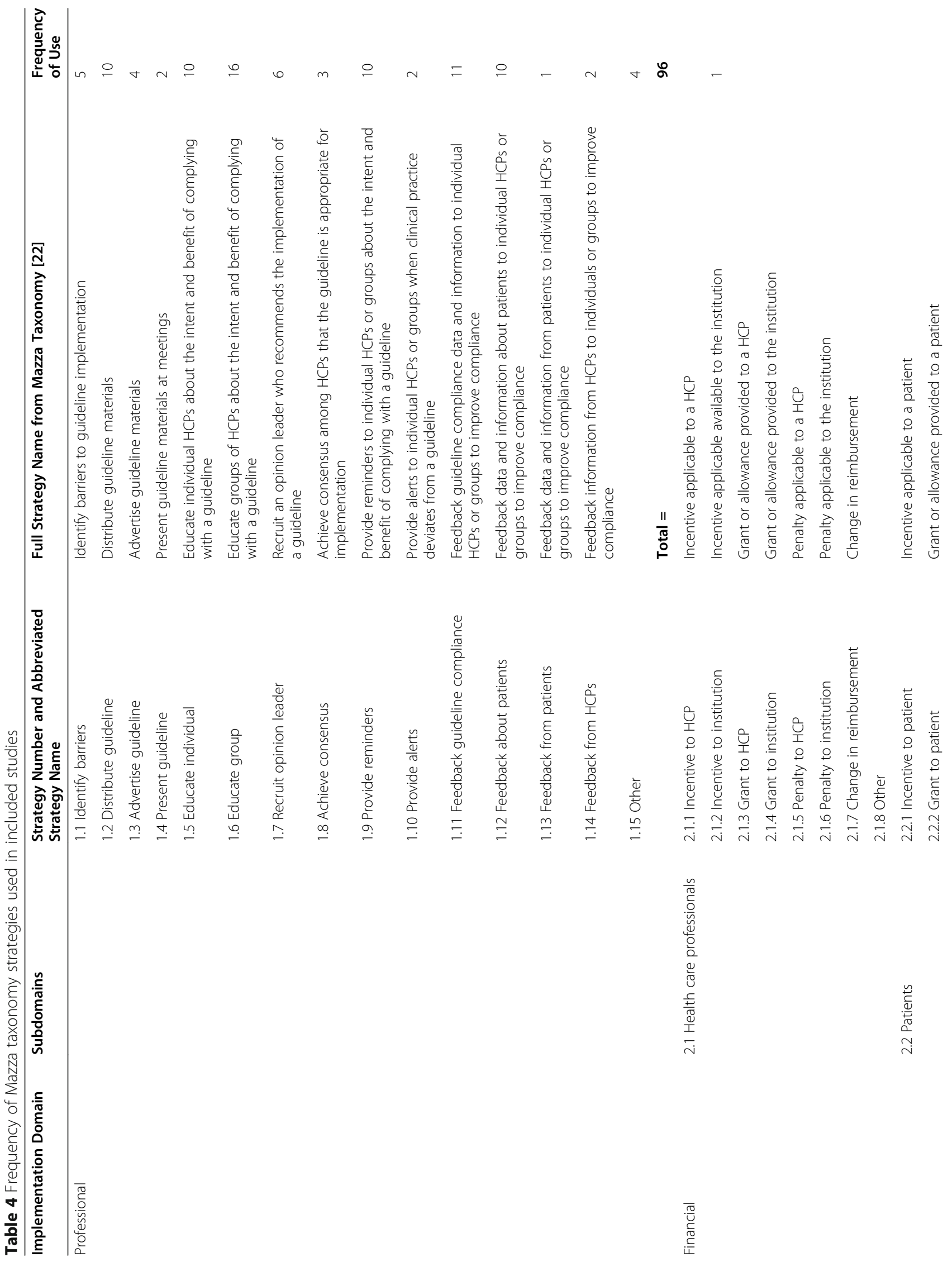




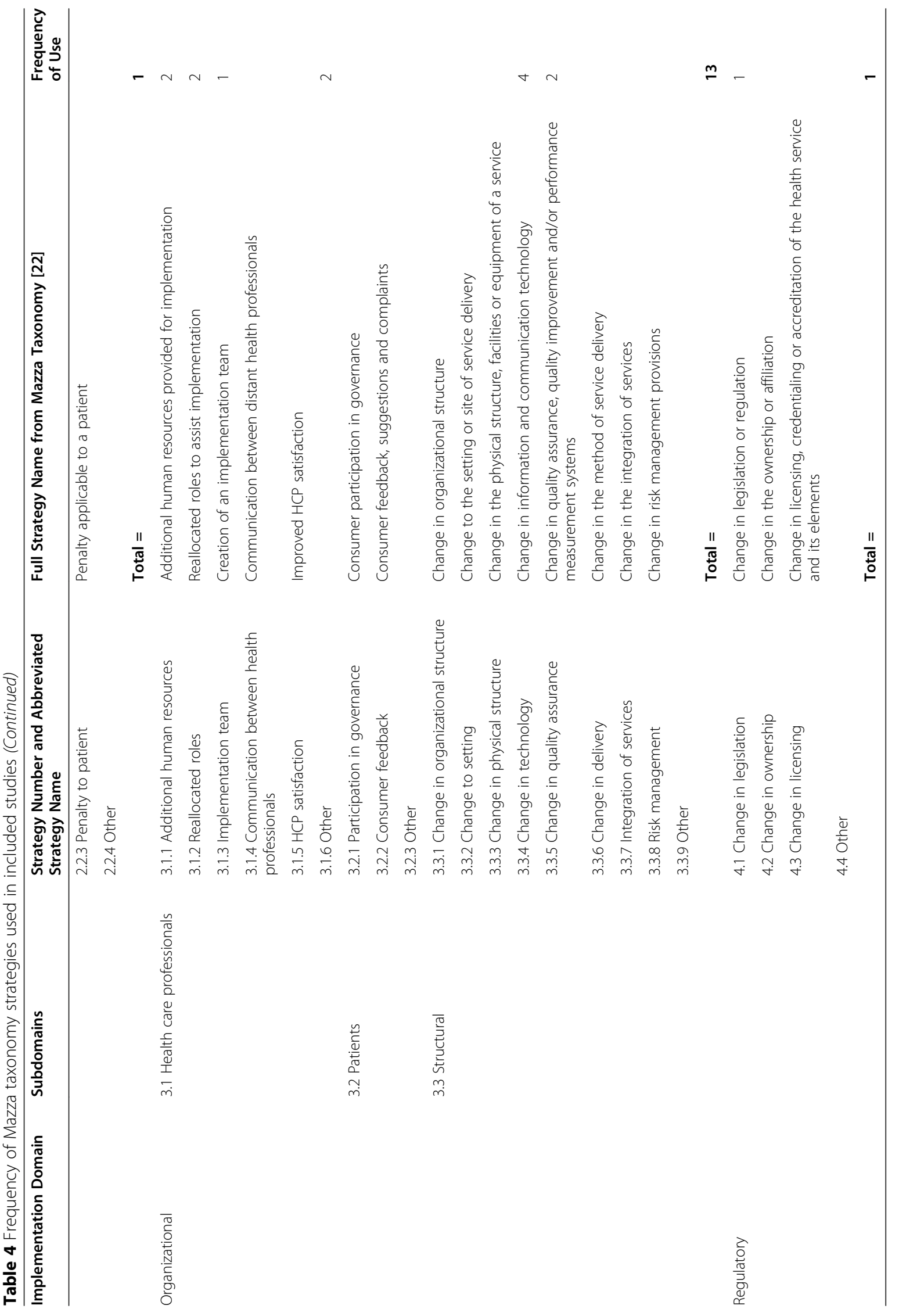

